\title{
Communication of Intent in Assistive Free Flyers
}

\author{
Daniel Szafir ${ }^{1}$, Bilge Mutluํㄹ ${ }^{1}$ and Terrence Fong ${ }^{2}$ \\ (1) University of Wisconsin-Madison, 1210 W. Dayton St., Madison, WI 53706 USA \\ (2) NASA Ames Research Center, Moffett Field, CA 94035 USA \\ \{dszafir,bilge\}@cs.wisc.edu; terry.fong@nasa.gov
}

\begin{abstract}
Assistive free-flyers (AFFs) are an emerging robotic platform with unparalleled flight capabilities that appear uniquely suited to exploration, surveillance, inspection, and telepresence tasks. However, unconstrained aerial movements may make it difficult for colocated operators, collaborators, and observers to understand AFF intentions, potentially leading to difficulties understanding whether operator instructions are being executed properly or to safety concerns if future AFF motions are unknown or difficult to predict. To increase AFF usability when working in close proximity to users, we explore the design of natural and intuitive flight motions that may improve AFF abilities to communicate intent while simultaneously accomplishing task goals. We propose a formalism for representing AFF flight paths as a series of motion primitives and present two studies examining the effects of modifying the trajectories and velocities of these flight primitives based on natural motion principles. Our first study found that modified flight motions might allow AFFs to more effectively communicate intent and, in our second study, participants preferred interacting with an AFF that used a manipulated flight path, rated modified flight motions as more natural, and felt safer around an AFF with modified motion. Our proposed formalism and findings highlight the importance of robot motion in achieving effective human-robot interactions.
\end{abstract}

\section{Categories and Subject Descriptors}

H.1.2 [Models and Principles]: User/Machine Systems—human factors, software psychology; H.5.2 [Information Interfaces and Presentation]: User Interfaces-evaluation/ methodology, usercentered design

\section{General Terms}

Design, Human Factors

\section{INTRODUCTION}

Free-flying robots hold great potential due to their unique abilities to freely traverse and survey environments. UAVs (unmanned aerial vehicles) or "drones", such as the General Atomics MQ-1 Predator and MQ-9 Reaper, are a staple in modern military operations [37], but do not interact in close proximity with people. An emerging class

Permission to make digital or hard copies of all or part of this work for personal or classroom use is granted without fee provided that copies are not made or distributed for profit or commercial advantage and that copies bear this notice and the full citation on the first page. Copyrights for components of this work owned by others than the author(s) must be honored. Abstracting with credit is permitted. To copy otherwise, or republish, to post on servers or to redistribute to lists, requires prior specific permission and/or a fee. Request permissions from permissions@ acm.org.

HRI' 14, March 3-6, 2014, Bielefeld, Germany.

Copyright is held by the owner/author(s). Publication rights licensed to ACM ACM 978-1-4503-2658-2/14/03 \$15.00.

http://dx.doi.org/10.1145/2559636.2559672

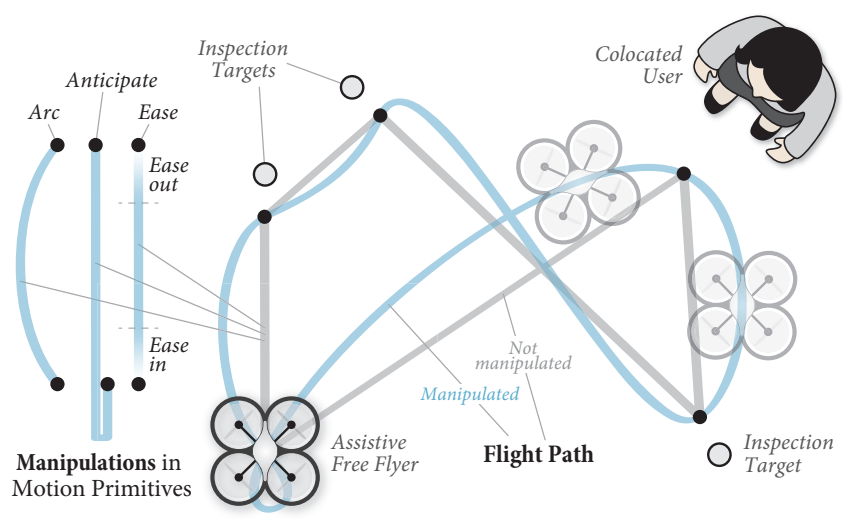

Figure 1: This work examines manipulations to the motion primitives that define the flight paths of free-flying robots, such as the quadrotor shown here, to more effectively express robot intent and increase usability.

of smaller aerial robots are envisioned to provide aid in domains including construction [16], utilities [44], search-and-rescue [15], and space $[8,9]$ by performing inspection, mapping, telepresence, and delivery tasks. We term these robots, which are represented in the growing body of research on micro air vehicles (MAVs) and multirotor aircraft [21, 32, 38], robotic airships [3, 6], and space exploration robots [9], "assistive free-flyers" (AFFs).

AFFs will require much greater collaboration and colocated interaction with humans due to the assistive nature of their tasks, and may eventually take the form of colocated social collaborators [30]. For example, NASA is currently developing an AFF called "Smart SPHERES," which is designed to be used inside spacecraft (e.g., the International Space Station) when humans are present to off-load routine work, increase astronaut productivity, and handle contingencies [9]. Smart SPHERES will perform a variety of mobile sensing tasks including environmental monitoring (e.g., air quality, radiation, sound levels), autonomous logistics management (e.g., inventory), and mobile camera work to support astronaut activities.

For AFFs such as the Smart SPHERES to successfully work and collaborate with colocated humans, designers must account for human perceptions of AFFs traveling within human environments. Although six-degrees-of-freedom (DOF) flight affords unique task abilities, users who do not generally interact with free-flying physical embodiments may not feel safe or may have trouble working near robots that exhibit unconstrained motion. Any potential misunderstandings regarding robot intentions may damage human-robot rapport, prove detrimental to task efficiency, reduce trust in automation, and may even be dangerous for the human collaborator. Alternatively, being able to understand robot intentions and predict where, when, how far, and how fast it will move may enable users to work and collaborate with AFFs more effectively. 
This work seeks to address the question of how designers might improve robot motions to increase the usability of AFFs that work near colocated humans. To this end, we developed a framework that deconstructs task-based AFF flight paths into a library of motion primitives and created a collection of parameterized manipulations inspired by animation principles to modify the trajectories and velocities of these primitives (Figure 1). We evaluated the utility of these modifications in expressing AFF intent in a formative exploration. Using our initial findings, we constructed modified AFF flight paths designed to express intent and support task requirements for a prototypical AFF interaction. A second, in-person experiment evaluated the effects of using these modified flight paths on high-level outcomes including usability and safety. Below, we outline relevant work that informed our design process and describe the development of our framework of primitives and motion modifications. Next, we describe the design, methodology, and results of both our formative exploration and in-person study. We conclude with a discussion highlighting the importance of considering robot motions for user interaction, especially for aerial robots with six DOF.

\section{RELATED WORK}

Our work draws from an emerging body of research focused on communicative robot motion. Additionally, our understanding of the design space is informed by research in film and computer animation, which offers a rich tradition of designing motions that are both communicative and visually appealing.

\subsection{Robot Motion and Intent}

When developing robots for human use, HRI researchers and designers must account for inferences users will make when perceiving robots in motion. Studies suggest that observers as young as sixand-a-half months will infer a mover's goals from both human and robot motion [17] and that robot motion can convey affective state $[34,38]$. Although prior work has examined the development of human-aware motions and intent-expressing gestures for robots with anthropomorphic and zoomorphic features [1, 13, 29, 40], it is not always clear how to apply these findings to robots, such as AFFs, lacking these morphological features.

For AFFs and other non-humanlike robots, manipulating motion trajectories appears to hold substantial promise in aiding users to better understand and predict robot intentions. Prior work has examined the effects of manipulating robot path planning, often using proxemics-based cost functions that prioritize paths respecting human spatial boundaries, on the physiological and subjective responses of colocated humans [18, 20, 23, 27, 28, 36]. Further research distinguishes robot motions that are legible, allowing users to correctly infer the robot's goal, and motions that are predictable, matching the expectations of an observer who knew the goal a priori [5, 26]. Dragan et al. [5] show that observers of videos in which robot trajectories were optimized for legibility, resulting in arced motions rather than straight lines, were better able to predict an end target. However, viewers who knew the target in advance reported a direct path, optimized for predictability, closely matched their expected trajectory, indicating that prior knowledge and expectations might confound the interpretation of goal-based trajectories.

Recent research has also examined robot motion beyond the realm of goal-based movement. For instance, research demonstrates that AFFs might be able to communicate affective states to users through manipulations of flight trajectories using the Laban Effort System [38]. Although motion seems to be a promising means of conveying affect, it may be challenging to specify such movements without the aid of a Laban-trained artist. Research using the ground-based iCat and Roomba robots has shown that both curvature and acceleration can have an effect on the robot's perceived affective state and can be generated algorithmically [34]. While such work is useful in designing motion that enables social robots to display varying levels of arousal, it does not inform us regarding the effects of such motions on user perceptions of robotic intent, trust, or competency. More work is needed to explore how robots, particularly those with freeflying abilities, might better express their intentions regarding where, when, how far, and how fast they will move.

\subsection{The Motion Design Space}

Film and computer animation research focuses on effective, visual communication of both affective state and intention. As such, prior research regarding the principles of animating characters may prove beneficial for designers interested in robot motions. Prior work in these fields offers eleven animation principles [22, 25]: squash and stretch, timing, anticipation, staging, follow through and overlapping action, straight ahead action and pose-to-pose action, slow in and out, arcs, exaggeration, secondary action, and appeal. Users find animations designed in accordance with these principles, for example squashing and stretching the shape of an animated bouncing ball to convey the ball's rigidity and mass, to be more appealing, and users are able to better predict virtual object trajectories when animation principles are applied [10].

Prior HRI research adapts certain animation techniques and principles to increase the naturalness of robot motions and expand their communicative abilities [11, 12, 13, 14, 33, 35, 40, 42, 43]. In one study, Takayama et al. [40] developed techniques based on anticipation and reaction principles to enable a robot to effectively express forethought and reactions to task outcomes. In another study, Gielniak and Thomaz [13] used an optimization approach to identify the most salient motion frame of a symbolic gesture and move this frame earlier in the motion, generating anticipation that increased observers' abilities to accurately perceive intent. Other research combines and blends animation principles to generate complex robot motions; for example, using anticipation, slow in and out, and secondary motion to create believable turning and sleeping motions for an iCat robot $[42,43]$ or using exaggeration and timing to exhibit nonverbal expression of emotions [33]. However, such research utilizes humanlike robots with limbs and expressive faces-features AFF's generally lack. Further investigation is needed exploring the potential benefits of adapting animation principles to the design space of robot motion for non-humanlike robots and investigating how low-level, easily implemented motion manipulations impact high-level human-robot interaction outcomes.

\section{FORMALIZATION AND MODELING}

To explore the design space of expressive motion for flying robots, we first developed a formal framework for AFF motion. This framework defines motion using three aspects and outlines a set of motion primitives that can be used to construct flight paths. These primitives represent a set of underlying actions that AFFs will undertake to complete tasks when operating near colocated humans, such as providing instruction or performing surveillance. Additionally, we developed a set of parameterized motion manipulations that might be applied to the primitives, which we evaluated for their ability to increase human perceptions of AFF intent and usability.

\subsection{Defining Motion}

We define AFF motion as a composition of three aspects: trajectory, velocity, and orientation. Trajectory and orientation are represented as three-dimensional vectors $T_{x y z}$ and $O_{x y z}$, which correspond to 
Table 1: We identified 11 primitives for AFFs working in environments with colocated humans.

\begin{tabular}{lll} 
Primitive & Category & Perspective \\
\hline Take off & Core & Exocentric \\
\hline Hover & Core & Exocentric \\
\hline Cruise & Core & Exocentric \\
\hline Land & Core & Exocentric \\
\hline Approach person & Interactive & Egocentric, Exocentric \\
\hline Avoid person & Interactive & Egocentric, Exocentric \\
\hline Depart person & Interactive & Egocentric, Exocentric \\
\hline Approach object & Interactive & Exocentric \\
\hline Avoid object & Interactive & Exocentric \\
\hline Depart object & Interactive & Exocentric \\
\hline Scan objects & Interactive & Exocentric \\
\hline
\end{tabular}

the position of an AFF and the direction it faces ${ }^{1}$, while velocity magnitude is represented by a scalar $V$.

\subsection{AFF Motion Primitives}

To explore the design space of expressive flight movements used while interacting with colocated operators, collaborators, and observers, we analyzed several hypothetical AFF interactions in scenarios including warehouse inspection, utilities surveillance, and telepresence in space missions that were inspired by prior AFF research. $[8,16,44]$. From a variety of potential AFF movements during such interactions, we developed a set of 11 motion primitives that could be combined to create all of the flight paths in our scenarios (Table 1). These primitives are divided into two main categories: (1) core principles of flight including taking off, landing, and hovering, and (2) interactive primitives such as approaching, avoiding, and departing that allow AFFs to work with objects and people in their environment. Additionally, primitives are classified using an understanding of viewer perspective as either egocentric, meaning the primitive represents AFF motion in a first-person interaction with the viewers themselves, or exocentric, meaning the motion represents AFF interaction with other objects or people in the environment viewed from a third-person perspective. These perspectives are adapted from work in robot perspective-taking [41] as well as teleoperation interfaces [7], which have shown the importance of considering viewer perspective in human-robot interactions. Certain primitives, such as approaching a person, can be both egocentric, as when an AFF approaches the viewer, and exocentric, as when an $\mathrm{AFF}$ approaches a second colocated human in the viewer's environment, and may be perceived differently across each perspective.

\subsection{Motion Manipulations}

Our design space for expressive motion encompasses all possible manipulations to AFF trajectories, velocities, and orientations while executing primitives. Within this space, the animation principles as well as previous work in robot motion literature guided us in devising several potential manipulations that might increase the saliency of AFF actions, including flight trajectories with arcs rather than straight lines, easing in and out of primitives using slow in and out velocity profiles, using anticipatory motions by first reversing trajectories for a short time, foreshadowing intentions by altering orientation to show directionality prior to movement, and applying exaggerated secondary motions such as side-to-side movements prior to completing the main trajectory. From these potential manipulations, we elected to explore arc trajectories, easing in and out of velocity profiles, anticipatory motions, and their combinations (Figure 2). Prior HRI research suggests that these manipulations

${ }^{1}$ Orientation assumes a front based on the side of the AFF with a camera. For AFFs with no obvious front, an arbitrary side can be chosen as long as it is kept consistent. may hold promise in communicating intent (e.g., optimized arc trajectories can improve motion legibility even for point robots [5]) and affect [34]. Further, these manipulations, when parameterized with respect to platform constraints, strongly parallel the underlying animation principles proposed to enhance human-robot interactions [42]. Below, we describe the development of each manipulation, followed by a study to evaluate the utility of these manipulations, based on principles of natural motion in articulated characters, in achieving intent-expressive AFF flight motion.

\subsubsection{Arcing}

Symmetric arcs between two points in three-dimensional space can be represented by considering the straight path between the points as a chord on a sphere. To generate an arc, we need only to specify the center point for the sphere by first constructing a midpoint $M$ between starting point $S$ and ending point $E$ and displacing $M$ by a desired amount which will correspond to the radius of the sphere:

$$
M=\frac{\omega(S+E)}{2}-R
$$

where $\omega=[0,2]$ defines how close $M$ is to the true midpoint of $S$ and $E$, and $R$ represents a vector $\langle x, y, z\rangle$ that displaces $M$ to define the depth and dimensionality of the curve. The choice of $R$ determines the shape of the arc; for example, $R_{x y z}=<0,-1,0>$ creates a vertical arc by specifying a spherical center below $S$ and $E$.

\subsubsection{Easing}

To generate easing, which we define as a velocity profile with slow in and out, we adapt previous work on slow-in and slow-out filters [24] and work on modifying velocity profiles to achieve smooth motion transitions for humanoid robots [12]. To ease into and out of desired velocities, we used a Gaussian function sampled at discrete time intervals:

$$
v(t)=a e^{-(x-\mu)^{2} / 2 \sigma^{2}}
$$

For this work, we assigned constant values to the Gaussian parameters as follows:

$$
a=\frac{1}{.5 \sqrt{2 \pi}}, \mu=0.5, \sigma=0.15
$$

to generate a smooth normalized curve in the range $[0,100]$ that can be scaled to desired velocities. By keeping track of flight distance (the area under the Gaussian curve), an AFF can use the first half of the curve to ease into the desired velocity and the second half of the curve when stopping, connecting the two halves of the curve with the desired maximum velocity (Figure 3 ).

\subsubsection{Anticipation}

Anticipatory actions, which can make movements more expressive, represent motion that occurs along the direction opposite to the

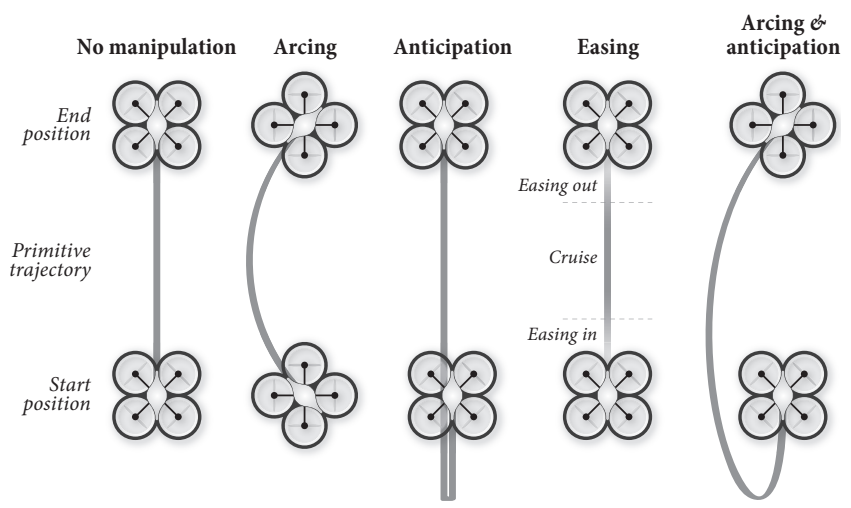

Figure 2: The arc, anticipation, and easing manipulations that we explored in this work and an example of a combined manipulation. 


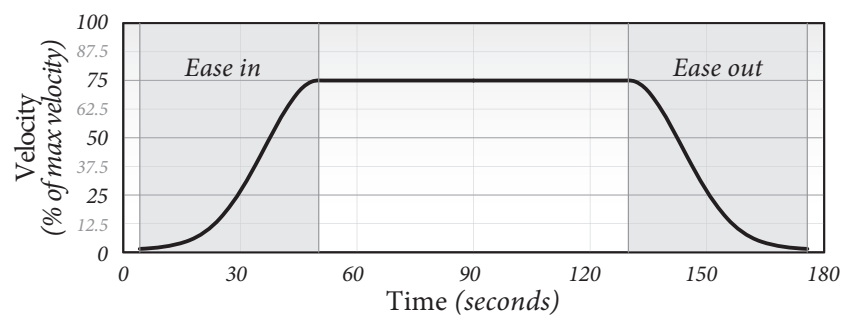

Figure 3: A velocity profile for an AFF using a Gaussian function to ease into and out of desired velocities.

main movement [19]. For AFFs, we define anticipatory motion as a trajectory vector $T$ :

$$
T=S+\theta(E-S)
$$

where $T$ is opposite of the direction of the main trajectory, defined as the start of the trajectory $S$ and end of the trajectory $E$, and is proportional in magnitude to the main trajectory by the scaling factor $\theta=[0,1]$. In this work, we used a constant $\theta=.3$, meaning that an AFF using anticipation would first fly approximately $1 / 3$ of the distance of its main trajectory in the opposite direction, then fly to the desired end point, lengthening the total trajectory by $33 \%$.

\section{FORMATIVE EXPLORATION}

To gain an understanding of how arcing, easing, and anticipatory AFF motion manipulations might impact viewers' abilities to perceive and reason about robot intent, we first conducted a formative study using pre-constructed videos of a virtual AFF. Due to the wholly unexplored design space of manipulating flight motions to showcase intent, we treated this study as a form of design exploration lacking a priori hypotheses. In the exploration, we gathered data on the effects of each potential motion manipulation on viewers' understanding of robot intent across a range of scenarios that sampled from each interactive AFF motion primitive.

\subsection{Study Design}

In our study, we examined the effects of manipulating the interactive AFF motion primitives identified in Section 3.2. We did not examine the effects of applying manipulations to core primitives due to their nature as actions necessary for basic flight. We created ten scenarios, one for each of the seven interactive primitives from an exocentric perspective and an additional three using an egocentric point of view for the primitives that can be viewed from both perspectives.

We used Unity ${ }^{2}$, a 3D game and animation engine, to develop the scenarios into short ( $\sim 10$ second) video clips showing a virtual AFF, based on a model of a commercial platform, moving throughout a realistic warehouse environment (Figure 4). To generate arc trajectories, we constructed smooth motion along the curves defined by the starting, ending, and center points using spherical linear interpolation (SLERP) [39]. To implement easing, velocities at each frame were sampled from the Gaussian velocity profile described

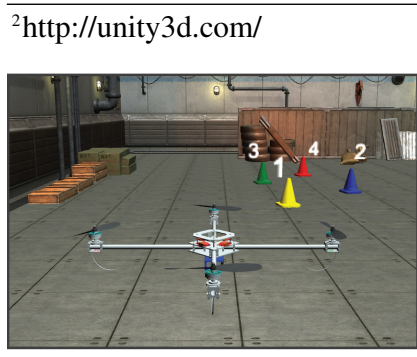

Depart person (Egocentric)

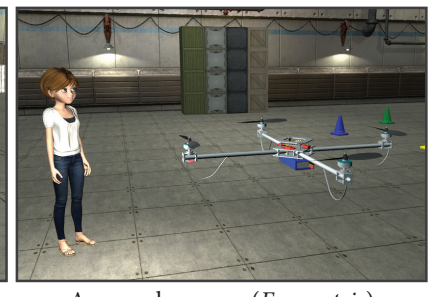

Approach person (Exocentric)
Figure 4: Formative exploration stimuli: an egocentric departure with four potential numbered targets and an exocentric approach. in Section 3.3.2 while keeping track of the distance that the AFF had traveled by approximating the area under the curve using trapezoidal Riemann sums. Eased velocity profiles were constructed such that the AFF used slow in and out for the first and last $15 \%$ of the main trajectory and traveled at the desired speed for the remaining $70 \%$. Anticipation was implemented as described as in Section 3.3.3 using $\theta=.3$; thus, a longer anticipation distance corresponded to a longer main trajectory. To limit potential confounding effects of orientation, the virtual AFF was designed without a distinct "front" and orientation was kept constant throughout each video.

We conducted a $2 \times 2 \times 2$ between-subjects experiment using our motion manipulations (arcing, easing, and anticipatory motion) as independent variables. Each manipulation had two levels corresponding to the manipulation either being present or absent. In total, we rendered 80 videos-each video corresponded to one of the 10 scenarios for each of the eight unique conditions made from all possible combinations of the presence/absence of arcing, easing, and anticipation with the parameters set at values described above. Each participant viewed 10 videos to see all primitives in a randomized order for a given condition. Following each video, users were presented with four potential options describing their belief regarding the robot's future movement intentions (e.g., for approach and avoid primitives, options included flying to the left, to the right, above, or stopping in front of the target). To limit potential learning effects, each participant first viewed a longer ( 30 second) video clip that showed the AFF exhibiting a longer flight path using the manipulations that corresponded to the participant's condition.

\subsection{Participants}

Participants were recruited from Amazon's Mechanical Turk infrastructure and randomly assigned to one of the eight conditions. As in previous HRI studies using Mechanical Turk (e.g., [5, 40]), we only recruited participants with at least a $95 \%$ approval rating and validated participant responses using a control question. In total, we recruited 85 participants (42 males and 43 females) who ranged in age from 18 to $80(M=35.29, S D=11.79)$. The entire task took participants on average 5 minutes, for which they were paid $\$ 1.00$.

\subsection{Measures}

We used three objective measures to evaluate the effects of our manipulations on participants' understanding of AFF intent: response time, accuracy, and a composite measure based on Guttman scoring $[2,5]$. To evaluate response time, participants were instructed to pause the video as soon as they could infer where they believed the robot was traveling. For example, in the video corresponding to an egocentric approach, participants were instructed before the video started that they should pause the video as soon as they believed they knew whether the robot would pass to their right, to their left, fly over them, or stop in front of them. Response time measured the time passed between when the video started and when participants paused the video. To evaluate accuracy, once users paused the video, the video disappeared, and participants filled out a four-item multiple-choice question. We created a composite measure based on Guttman structures that penalized wrong answers and assigned scores to correct answers based on the response-time measure.

\subsection{Results and Discussion}

We analyzed the objective measurements using a one-way analysis of variance (ANOVA) with the main experimental manipulations of arcing, easing, and anticipation, and all two-way and three-way interactions among these manipulations, as a fixed effects. Dunnett's test controlled for Type I errors in comparing all individual manipulations and their combinations against a no manipulation 
Table 2: Results illustrate the most effective motion manipulations compared with a no-manipulation baseline on accuracy, response time, and a composite measure. Bold text indicates marginal and significant results. $\left(^{\circ}\right),(+)$, and $(-)$ denote perfect accuracy, increased scores, and decreased scores, respectively.

\begin{tabular}{|c|c|c|c|c|c|c|c|}
\hline \multirow[b]{2}{*}{ Primitive } & \multirow[b]{2}{*}{ Perspective } & \multicolumn{3}{|l|}{ Best Manipulation } & \multicolumn{3}{|c|}{ Main Effects on Composite Score } \\
\hline & & Accuracy & Response & Composite Score & Arc & Ease & Anticipate \\
\hline Approach person & Egocentric & Ease $^{\circ}$, anticipate ${ }^{\circ}$, ease+anticipate ${ }^{\circ}$ & Arc & Ease & $F(1,77)=2.26 p=.137$ & $F(1,77)=0.004 p=.947$ & $F(1,77)=2.91 p=.092(-)$ \\
\hline Avoid person & Egocentric & Arc $^{\circ}$, arc + ease $e^{\circ}$, arc+ease+anticipate ${ }^{\circ}$ & None & Ease $(p=.028)$, arc + ease $(p=.003)$ & $F(1,77)=1.73, p=.193$ & $F(1,77)=5.76, p=.019(+)$ & $F(1,77)=1.90, p=.172$ \\
\hline Depart person & Egocentric & Ease, none ${ }^{\circ}$ & Ease & Ease $(p=.075)$ & $F(1,77)=11.70, p=.001(-)$ & $F(1,77)=13.62, p<.001(+)$ & $F(1,77)=11.93, p<.001(-)$ \\
\hline Approach person & Exocentric & Ease+anticipate $^{\circ}$ & None & None & $F(1,77)=.577, p=.450$ & $F(1,77)=5.45, p=.022(-)$ & $F(1,77)=0.060, p=.807$ \\
\hline Avoid person & Exocentric & Arc, arc+ease ${ }^{\circ}$ & Ease & Arc+ease $(p=.002)$ & $F(1,77)=3.09, p=.083(+)$ & $F(1,77)=1.16, p=.286$ & $F(1,77)=10.28, p=.002(-)$ \\
\hline Depart person & Exocentric & Ease+anticipate & Ease & Ease & $F(1,77)=1.06, p=.306$ & $F(1,77)=4.01, p=.049(+)$ & $F(1,77)=3.28, p=.075(-)$ \\
\hline Avoid object & Exocentric & Arctease & Ease & Ease $(p=.070)$ & $F(1,77)=0.013, p=.911$ & $F(1,77)=0.295, p=.589$ & $F(1,77)=3.56, p=.063(-)$ \\
\hline Depart object & Exocentric & Arc+ease+anticipate & Arc+ease & Arctease & $F(1,77)=0.956, p=.331$ & $F(1,77)=20.72, p<.0001(+)$ & $F(1,77)=4.70, p=.033(-)$ \\
\hline Scan objects & Exocentric & Ease+anticipate & Ease & Ease+anticipate & $F(1,77)=0.001, p=.982$ & $F(1,77)=0.855, p=.358$ & $F(1,77)=0.634, p=.428$ \\
\hline All & N/A & Ease+anticipate $(p=.071)$ & Ease & Ease & $F(1,77)=2.34, p=.131$ & $F(1,77)=7.20, p=.009(+)$ & $F(1,77)=13.66, p<.001(-)$ \\
\hline
\end{tabular}

baseline. Contrast tests using Scheffé's method analyzed the relative contributions of each manipulation.

Table 2 provides a summary of our results. Although we found no manipulation that significantly improved accuracy over the baseline for any individual primitive, several manipulations led to $100 \%$ accuracy rates across a very diverse population of online participants. Across all primitives, we found that a combination of easing and anticipation marginally increased accuracy over baseline motions $(p=.071)$, demonstrating the potential of these manipulations in accurately expressing intent.

We analyzed our composite measure to gain a better understanding of what manipulations might improve both accuracy and response time. We found that an AFF using both arcing and easing motions when avoiding a person significantly outperformed baseline motions from both the egocentric $(p=.003)$ and exocentric perspective $(p=.002)$. Further, we found that easing motions marginally outperformed the baseline when departing a person from an egocentric perspective $(p=.075)$. These results indicate that smooth velocities may be particularly important when closely interacting with users and that arcing may effectively signal that an AFF will not approach or collide with a user in a manner similar to hallway encounters with ground robots [31].

To further understand the effects of our manipulations, we analyzed the relative individual contributions of arcing, easing, and anticipation to the composite measure. We found that anticipation significantly lowered composite scores for several primitives; however, as these negative effects were not reflected in the accuracy measure, we believe the poor performance of anticipation in the composite measure may be due to slower response times caused by the increased trajectory lengths from adding anticipatory motions. These findings indicate a potential trade-off between how quickly and how accurately users are able to predict robot intent, and that each primitive and each combination of primitives may provide a different balance of this trade-off. We found that easing helped in a number of primitives, especially in the egocentric perspective where it significantly outperformed manipulations lacking easing for avoidance, $F(1,77)=5.76, p=.019$, and departure, $F(1,77)$ $=13.62, p<.001$, providing further evidence that smooth velocity profiles are beneficial for AFFs operating near humans.

\section{COMMUNICATIVE FLIGHT PATHS}

Our formative exploration regarding the use of arcing, easing, and anticipatory motions to increase viewer understandings of AFF intent revealed that these manipulations appeared to positively effect viewers' understandings of robot intent for certain AFF primitives. To gain a further understanding of how such low-level motion manip- ulations might effect high-level colocated interactions with AFFs, we constructed complete, task-based AFF flight paths informed by the results from our formative exploration. These flight paths were created by blending several primitives, each of which used the motion manipulation that had achieved the highest composite score in our initial study. We then designed and conducted an inperson laboratory experiment in which participants interacted with a physically-embodied AFF to examine participant responses to an AFF using communicative flight primitives.

\subsection{Hypotheses}

We developed several hypotheses seeking to capture high-level interaction outcomes for participants observing a colocated AFF using flight paths composed from primitives with the arcing, easing, and anticipatory motion manipulations that our design exploration found to be most effective compared with non-manipulated primitives:

Hypothesis 1. Colocated individuals will prefer working with an AFF using manipulated flight paths than baseline paths.

Hypothesis 2. Colocated individuals will view manipulated AFF flight paths to be more natural and intuitive than baseline motions.

Hypothesis 3. Colocated individuals will feel safer interacting with an AFF using manipulated flight paths than baseline paths.

\subsection{Study Design and Procedure}

We designed a $2 \times 1$ within-participants study to evaluate participant responses when observing a physically embodied, colocated AFF executing a flight path designed to express intent. Our independent variable represented whether the AFF flight path was composed from primitives with or without the manipulations that scored highest in the composite measure from our online study, involving two levels: motion manipulations present and absent. Dependent variables included participants' ratings regarding the presence of the manipulations across conditions, robot usability, motion naturalness, and how safe they felt near the AFF.

During the experiment, the AFF completed two flight paths scanning several QR codes placed within the experimental environment. Each flight corresponded to one of the experimental conditions. Both flight paths contained the same number and type of primitives (Table 3), and the order in which the flight paths and experimental conditions were presented was counterbalanced. To implement a flight path, the experimenter activated pre-programmed primitives using custom Java code to send pitch, roll, yaw, and elevation commands during the experiment (e.g., hover, approach person), which were created both with and without motion manipulations. The flight path was controlled in this Wizard-of-Oz (WoZ) manner to ensure participant safety and to correct for any path errors. 
Table 3: The primitives and the motion manipulations used in each condition of our in-person study.

\begin{tabular}{rl|l|l|} 
Primitive & Perspective & Absent & Present \\
\hline Take off & Exocentric & None & None \\
Hover & Exocentric & None & None \\
\hline Cruise & Exocentric & None & None \\
\hline Land & Exocentric & None & None \\
\hline Approach person & Egocentric & None & Ease \\
\hline Avoid person & Exocentric & None & Arc+ease \\
\hline Depart person & Egocentric & None & Ease \\
\hline Approach object & Exocentric & None & Anticipate \\
Depart object & Exocentric & None & Arc+ease \\
\hline
\end{tabular}

We used the Parrot AR.Drone $2.0^{3}$ as our AFF platform. Unlike the virtual AFF in the formative exploration, the AR.Drone has a distinct front with a forward-facing camera. To limit potential confounding effects of orientation, in both conditions, the operator always rotated the AFF to achieve an orientation facing the current target prior to executing primitives. To simplify arc implementation, we simultaneously varied the velocity commands for two of the pitch, roll, yaw, and elevation components by monotonically increasing one dimension while monotonically decreasing another such that the components always summed to one, generating a quarter arc with $\omega=1$. Arcs were implemented such that the AFF arced vertically over the person for the "avoid person" primitive and best aligned the AFF with its next target for the "depart object" primitive. Easing was accomplished by sending velocity commands with magnitudes approximating our Gaussian curve; however, due to sensor limitations in tracking AFF travel distance, the ease in and out effects were scaled to take 1.5 seconds each instead of being based on distance traveled as in our formative exploration. Anticipation distances, used only for approaching objects, were pre-measured based on the experimental setup using $\theta=.3$, as previously described.

The experimental procedure took roughly 30 minutes and consisted of six main phases: (1) introduction, (2) observation, (3) evaluation, (4) observation, (5) evaluation, and (6) conclusion. First, the experimenter obtained informed consent and introduced the participant to an experimental confederate acting as a fellow participant. In reality, the confederate's presence allowed participants to view AFF movements around another person from an exocentric perspective. Participants and the confederate, seated at tables on opposite sides of a controlled room, were instructed to act as quality control personnel by evaluating two operators flying an AFF and identifying which QR codes each operator targeted during their flight (Figure 5). QR codes were placed throughout the environment, including in front of, above, and to the sides of both the participant and the confederate. In phases 2 and 3, participants observed an AFF execute a

${ }^{3}$ http://ardrone2.parrot.com/

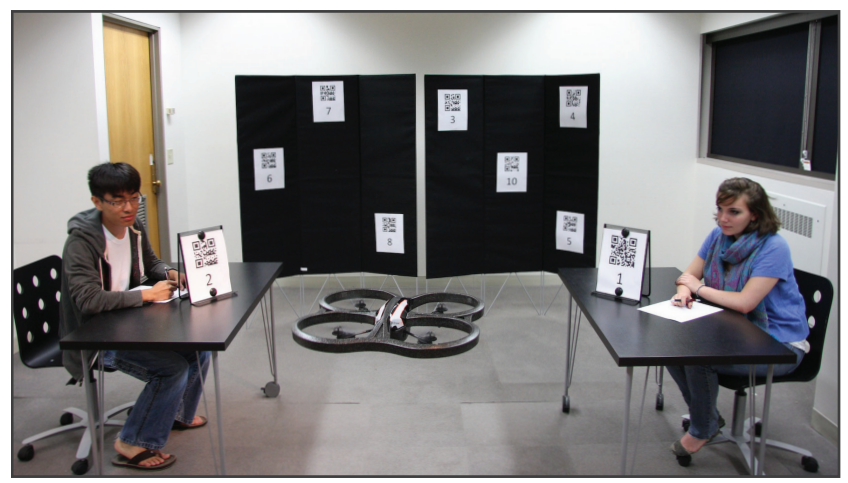

Figure 5: A participant (right) and a confederate in our experiment observe an AFF as it scans QR codes in their environment. flight path that was controlled in the WoZ manner described above and completed a questionnaire evaluating their experience and the "operator" whom they believed had just flown the AFF. Phases 4 and 5 repeated these steps, with participants viewing and evaluating a second flight path under control of a second "operator." In phase 6 , the experimenter collected demographic information, debriefed the participant, and paid them $\$ 5.00$ for their time.

\subsection{Participants}

We recruited a total of 24 native-English-speaking participants (12 males and 12 females) ${ }^{4}$ from the University of Wisconsin-Madison campus. Average participant age was $20.96(S D=2.17)$, with a range of 18-27. On a seven-point scale, participants reported a moderate prior familiarity with robots $(M=3.04, S D=1.41)$, but a low familiarity with aerial robots $(M=2.58, S D=1.54)$. Participants reported a wide range of occupations and majors, including 17 distinct areas of study.

\subsection{Measurement and Analysis}

Dependent variables were captured through a number of scales administered as a questionnaire. We constructed scales to confirm that participants noticed the differences in motion manipulations between conditions ( 6 items, Cronbach's $\alpha=.708$ ) and to evaluate participant ratings of usability for each AFF "operator" (4 items, Cronbach's $\alpha=.824$ ), the naturalness of the AFF motion ( 5 items, Cronbach's $\alpha=.910$ ), and how safe the participants felt interacting with the AFF ( 3 items, Cronbach's $\alpha=.746$ ). We utilized a one-way repeated-measures analysis of covariance (ANCOVA) with the main experimental manipulation as a fixed effect, participant gender and the order of the presented flight paths as covariates, and participant ID as a random effect to analyze the data from our manipulation checks and dependent variables.

\subsection{Results}

We verified manipulation success and found that participants correctly perceived the AFF's use of arcing, easing, and anticipation between conditions, $F(1,23)=12.00, p=.002$. We performed a post-hoc power analysis for each dependent variable. On the basis of our population size, the observed standard deviations, and a medium expected effect size $(\delta=.5)$ [4], we found power levels of $.838, .518$, and .811 for usability $(\sigma=1.15)$, motion naturalness $(\sigma=1.69)$, and safety $(\sigma=1.19)$, respectively.

Hypothesis 1 predicted that participants would prefer working with an AFF that utilized flight paths with the most effective arcing, easing, and anticipation manipulations from our formative study. To test this hypothesis, we analyzed participants' ratings of each AFF "operator," using items on whether participants would want to work with the operator again, how well they believed the operator performed, and their confidence in the operator's control of the AFF. Participants significantly preferred the "operator" whose flight path used our manipulations, $F(1,23)=13.39, p=.001$.

Hypothesis 2 predicted that participants would find AFF flight paths composed from manipulated primitives to be more natural and smooth. An analysis on participant perceptions regarding the AFF's motion supported this hypothesis. Participants found manipulated paths to be significantly more natural, smooth, elegant, graceful, and intuitive, $F(1,23)=5.24, p=.032$.

Hypothesis 3 predicted that participants would feel safer working near an AFF executing flight paths with motions designed to convey intent. Our results provide support for this hypothesis, with participants rating the AFF that used primitives with motion manipulations as more safe and in control, $F(1,23)=5.18, p=.033$.

${ }^{4} \mathrm{~A}$ post-hoc power analysis is included in Section 5.5. 


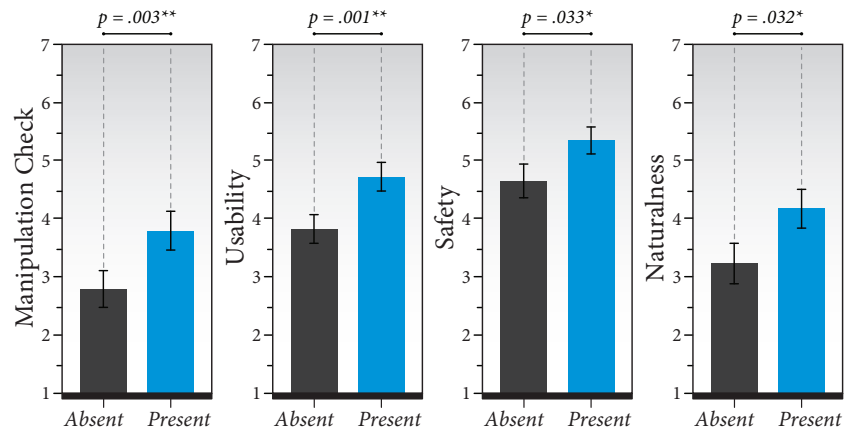

Figure 6: Results show that our manipulations positively effected participants' ratings of AFF usability, motion naturalness, and their sense of safety. $\left(^{*}\right)$ and $\left({ }^{* *}\right)$, denote $p<.05$ and $p<.01$, respectively.

Figure 6 summarizes the major results of the study. Only marginal and significant effects are reported.

\section{DISCUSSION}

Hypothesis 1 predicted that individuals would prefer working with a colocated AFF that communicated its flight path intentions using motion. Our results confirmed Hypothesis 1, showing how lowlevel manipulations to motion primitives significantly increased the usability ratings of a colocated AFF. Our results also confirmed Hypotheses 2 and 3; participants rated manipulated flight motions as more natural and felt safer near an AFF using a manipulated flight path. The flight paths that participants found to be effective were constructed using the manipulations that our formative study showed to best express intent, demonstrating a potential link between the animation principles that inspired the manipulations, the expression of intent demonstrated in our formative study, and the improvement in usability found in our in-person experiment. However, while our findings highlight the promise of applying a limited set of animation principles to aerial robot motions to improve colocated interaction outcomes, future work is needed to further explore the choice of manipulations, identify optimal manipulation parameters, and explore additional methods of conveying intent.

Manipulation Considerations. Although our manipulations proved effective, we consider our work to be a preliminary exploration of the rich design space offered by manipulating AFF motions. Our formative exploration provided evidence that arcing, easing, and anticipation manipulations, inspired by the principles of natural motion and previous research in expressive robot motion, may also be beneficial in expressing intent. However, there are many other potential manipulations, such as modifying orientations or adding secondary actions, that might better convey intent, minimize energy constraints, or express other AFF properties. Our design focused on manipulating the motions of the interactive primitives, which appear most suited for communicating intent and can be reasonably manipulated without disrupting the core actions necessary to basic flight. Future explorations might examine manipulations to core primitives as well, for example modifying hovering behaviors to designate internal states such as idle or engaged.

Parameter Considerations. In choosing parameter values for real-world settings, designers must be aware of several considerations that may impact manipulation effectiveness. Environmental factors may constrain certain choices; in real-world interactions, choosing an improper $R_{x y z}$ while arcing or too great a $\theta$ while performing anticipatory motion could lead to collisions with obstacles. Easing may not be appropriate or noticeable for very short distances or may result in inefficiencies for longer flights. For longer distances, the proportion of the trajectory using easing may be more effectively constrained using flight time, as we did in our in-person study, rather than distance. Parameter choices themselves may influence observer understanding regarding robot intent; for example, varying $R_{x y z}$ values will alter arc shapes, potentially leading to different interpretations by viewers (e.g., people may prove more adept at interpreting vertical rather than horizontal arcs as avoidance). More work is needed to investigate how parameter choices might be optimized and adjusted to environmental constraints.

Platform Considerations. Although we controlled for orientation in our experiment, our AFF platform contains secondary motions due to the physical nature of its flight that may have confounded participants' abilities to assess AFF intent. Our platform, as with any quadrotor, adjusts pitch, roll, and yaw to navigate; such changes may themselves provide anticipatory movements telegraphing motion intent. As these secondary motions are proportional to acceleration, flight paths lacking a smooth velocity profile had more noticeable secondary movements, possibly reducing the effect size between our conditions. Future work might explore the potential confounding nature of such secondary movements as well as test the value of the motion manipulations on additional platforms with alternative form factors and constraints.

Additional Methods to Convey Intent. There are several other potential methods that might be used to convey AFF intent. Future work might explore orientation, which might act as a gaze mechanism in providing clues to robot intentions for AFFs with an obvious front. Alternatively, designers might utilize multimodal feedback such as LEDs or other signaling mechanisms prior to trajectory changes. Future work might address challenges such as designing signaling mechanisms for unconstrained six DOF movement, resolving issues in signal occlusion, and comparing the relative effectiveness of various signaling approaches. Communicating intentions quickly and unambiguously is a critical challenge for AFFs. Integrating communicative motions, orientations, and signaling mechanisms may ultimately prove the most effective solution.

\subsection{Research and Design Implications}

This research sought to explore the design of effective motions that might increase colocated AFF usability. Our work has important theoretical, methodological, and practical implications for researchers and developers seeking to bring AFFs into human environments. Our motion compositions, operationalized in a laboratory experiment, showcase the potential of subtle motion manipulations in eliciting high-level interaction outcomes, including participant desire to work near an AFF and sense of safety, and highlight a potential link between natural and intent-expressive motion. Our AFF motion framework outlines a design space for modifying the trajectory, velocity, and orientation profiles of AFF primitives to achieve outcomes such as conveying intent. Our design methodology demonstrates an effective strategy for examining the potential of various motion manipulations and illustrates how research in other relevant areas such as computer animation can inspire the design of effective human-robot interaction. Finally, this research might inform future explorations with AFFs by providing a model for AFF experimental scenarios and demonstrating practical manipulations that are shown to improve the reactions of colocated participants.

\section{CONCLUSION}

In this work, we sought to explore how designers might improve robot motions to increase the usability of free-flying robots acting in human environments. After analyzing the motion design space by deconstructing high-level flight paths into composite primitives, we developed several parameterized manipulations to the motions of these primitives, inspired by computer and film animation principles. In a formative exploration using a virtual AFF, we investigated the usefulness of these manipulations in conveying intent. Our find- 
ings informed the design of manipulations to primitive motions for a physically embodied AFF to use while executing task-based flight paths. We evaluated these flight paths in an in-person experiment, and found that our motion designs significantly improved participants' preferences for working with an AFF, ratings of motion naturalness, and sense of safety, demonstrating the potential of designing flight motions to enhance user interactions.

\section{ACKNOWLEDGMENTS}

This research was supported by a NASA Space Technology Research Fellowship under award NNX12AN14H. We thank Mark Micire, Brandi Hefty, Ross Luo, Catherine Steffel, and Danielle Albers for their assistance in this research.

\section{REFERENCES}

[1] T. Asfour and R. Dillmann. Human-like motion of a humanoid robot arm based on a closed-form solution of the inverse kinematics problem. In Proc IROS'03, volume 2, pages 1407-1412, 2003.

[2] G. R. Bergersen, J. E. Hannay, D. I. Sjoberg, T. Dyba, and A. Karahasanovic. Inferring skill from tests of programming performance: Combining time and quality. In Proc ESEM'11, pages 305-314, 2011

[3] S. S. Bueno, J. R. Azinheira, J. Ramos Jr, E. C. d. Paiva, P. Rives, A. Elfes, J. R. Carvalho, G. F. Silveira, et al. Project aurora: Towards an autonomous robotic airship. In Proc IROS'02., pages 43-54, 2002.

[4] J. Cohen. Statistical power analysis for the behavioral sciencies. Routledge, 1988.

[5] A. D. Dragan, K. C. Lee, and S. S. Srinivasa. Legibility and predictability of robot motion. In Proc HRI'13, pages 301-308, 2013.

[6] A. Elfes, S. Siqueira Bueno, M. Bergerman, and J. Ramos Jr. A semi-autonomous robotic airship for environmental monitoring missions. In Proc ICRA'98., volume 4, pages 3449-3455, 1998.

[7] F. Ferland, F. Pomerleau, C. T. Le Dinh, and F. Michaud. Egocentric and exocentric teleoperation interface using real-time, $3 \mathrm{~d}$ video projection. In Proc HRI'09, pages 37-44, 2009

[8] T. Fong, R. Berka, M. Bualat, M. Diftler, M. Micire, D. Mittman, V. SunSpiral, and C. Provencher. The human exploration telerobotics project. In Proc Global Space Exploration Conference, 2012.

[9] T. Fong, M. Micire, T. Morse, E. Park, C. Provencher, V. To, D. Wheeler, D. Mittman, R. J. Torres, and E. Smith. Smart spheres: a telerobotic free-flyer for intravehicular activities in space. In Proc. AIAA Space'13, 2013.

[10] M. Garcia, J. Dingliana, and C. O'Sullivan. Perceptual evaluation of cartoon physics: accuracy, attention, appeal. In Proc. APGV'08, pages 107-114, 2008.

[11] M. J. Gielniak, C. K. Liu, and A. L. Thomaz. Secondary action in robot motion. In IEEE RO-MAN'10, pages 310-315, 2010.

[12] M. J. Gielniak, C. K. Liu, and A. L. Thomaz. Stylized motion generalization through adaptation of velocity profiles. In IEEE RO-MAN'10, pages 304-309, 2010

[13] M. J. Gielniak and A. L. Thomaz. Anticipation in robot motion. In IEEE RO-MAN'11, pages 449-454, 2011.

[14] M. J. Gielniak and A. L. Thomaz. Enhancing interaction through exaggerated motion synthesis. In Proc HRI'12, pages 375-382, 2012.

[15] M. A. Goodrich, B. S. Morse, D. Gerhardt, J. L. Cooper, M. Quigley, J. A. Adams, and C. Humphrey. Supporting wilderness search and rescue using a camera-equipped mini uav. Journal of Field Robotics, 25(1-2):89-110, 2008.

[16] J. Irizarry, M. Gheisari, and B. N. Walker. Usability assessment of drone technology as safety inspection tools. Journal of ITcon'12, 17:194-212, 2012

[17] K. Kamewari, M. Kato, T. Kanda, H. Ishiguro, and K. Hiraki. Six-and-a-half-month-old children positively attribute goals to human action and to humanoid-robot motion. Cognitive Development, 20(2):303-320, 2005.

[18] H. Kidokoro, T. Kanda, D. Brscic, and M. Shiomi. Will I bother here? A robot anticipating its influence on pedestrian walking comfort. In Proc HRI'13, pages 259-266, 2013.
[19] J.-H. Kim, J.-J. Choi, H. J. Shin, and I.-K. Lee. Anticipation effect generation for character animation. In Advances in Computer Graphics, pages 639-646. 2006.

[20] R. Kirby. Social Robot Navigation. PhD thesis, Robotics Institute, Carnegie Mellon University, Pittsburgh, PA, May 2010.

[21] T. Krajník, V. Vonásek, D. Fišer, and J. Faigl. Ar-drone as a platform for robotic research and education. In Research and Education in Robotics-EUROBOT 2011, pages 172-186. 2011.

[22] J. Krasner. Motion graphic design: applied history and aesthetics. Focal Press, 2013.

[23] D. Kulic and E. Croft. Physiological and subjective responses to articulated robot motion. Robotica, 25(01):13-27, 2007.

[24] J.-Y. Kwon and I.-K. Lee. An animation bilateral filter for slow-in and slow-out effects. Graphical Models, 73(5):141-150, 2011.

[25] J. Lasseter. Principles of traditional animation applied to $3 \mathrm{~d}$ computer animation. In Proc SIGGRAPH'87, volume 21, pages 35-44, 1987.

[26] C. Lichtenthaler, T. Lorenz, M. Karg, and A. Kirsch. Increasing perceived value between human and robots - measuring legibility in human aware navigation. In Proc ARSO'12, pages 89-94, 2012.

[27] J. Mainprice, E. Akin Sisbot, L. Jaillet, J. Cortés, R. Alami, and T. Siméon. Planning human-aware motions using a sampling-based costmap planner. In Proc ICRA'11, pages 5012-5017, 2011.

[28] J. Mainprice, M. Gharbi, T. Siméon, and R. Alami. Sharing effort in planning human-robot handover tasks. In IEEE RO-MAN'12, pages 764-770, 2012

[29] C. L. Nehaniv, K. Dautenhahn, J. Kubacki, M. Haegele, C. Parlitz, and R. Alami. A methodological approach relating the classification of gesture to identification of human intent in the context of human-robot interaction. In IEEE RO-MAN'05, pages 371-377, 2005.

[30] W. S. Ng and E. Sharlin. Collocated interaction with flying robots. In IEEE RO-MAN'11, pages 143-149, 2011.

[31] E. Pacchierotti, H. I. Christensen, and P. Jensfelt. Human-robot embodied interaction in hallway settings: a pilot user study. In IEEE $R O$-MAN'05, pages 164-171, 2005.

[32] K. Pfeil, S. L. Koh, and J. LaViola. Exploring 3d gesture metaphors for interaction with unmanned aerial vehicles. In Proc IUI'13, pages 257-266, 2013.

[33] T. Ribeiro and A. Paiva. The illusion of robotic life: principles and practices of animation for robots. In Proc HRI'12, pages 383-390, 2012.

[34] M. Saerbeck and C. Bartneck. Perception of affect elicited by robot motion. In Proc HRI'10, pages 53-60, 2010.

[35] M. Saerbeck and A. J. van Breemen. Design guidelines and tools for creating believable motion for personal robots. In IEEE RO-MAN'O7, pages 386-391, 2007.

[36] L. Scandolo and T. Fraichard. An anthropomorphic navigation scheme for dynamic scenarios. In Proc ICRA'11, pages 809-814, 2011.

[37] N. Sharkey. Death strikes from the sky: the calculus of proportionality. Technology and Society Magazine, IEEE, 28(1):16-19, 2009.

[38] M. Sharma, D. Hildebrandt, G. Newman, J. E. Young, and R. Eskicioglu. Communicating affect via flight path exploring use of the laban effort system for designing affective locomotion paths. In Proc HRI'13, pages 293-300, 2013.

[39] K. Shoemake. Animating rotation with quaternion curves. In Proc SIGGRAPH'85, volume 19, pages 245-254, 1985.

[40] L. Takayama, D. Dooley, and W. Ju. Expressing thought: improving robot readability with animation principles. In Proc HRI'11, pages 69-76, 2011

[41] J. G. Trafton, N. L. Cassimatis, M. D. Bugajska, D. P. Brock, F. E Mintz, and A. C. Schultz. Enabling effective human-robot interaction using perspective-taking in robots. Systems, Man and Cybernetics, IEEE Trans on, 35(4):460-470, 2005.

[42] A. Van Breemen. Bringing robots to life: Applying principles of animation to robots. In Proc CHI'04, 2004.

[43] A. J. van Breemen. Animation engine for believable interactive user-interface robots. In Proc IROS'04, volume 3, pages 2873-2878, 2004.

[44] B. Wang, X. Chen, Q. Wang, L. Liu, H. Zhang, and B. Li. Power line inspection with a flying robot. In Proc CARPI'10, pages 1-6, 2010. 